\title{
Vitaminas antioxidantes e prevenção da arteriosclerose na infância
}

\author{
Antioxidant vitamins and prevention of atherosclerosis in childhood
}

Adriana Boni ${ }^{1}$ Camila Pugliese ${ }^{2}$, Cristiane Chiantelli Cláudio ${ }^{2}$, Rose Vega Patin ${ }^{3}$, Fernanda Luísa C. Oliveira ${ }^{4}$

\section{RESUMO}

Objetivo: Revisar os potenciais efeitos antioxidantes das vitaminas A, C e E na prevenção do desenvolvimento da arteriosclerose na infância, com ênfase na prevenção da dislipidemia.

Fontes de dados: Pesquisa bibliográfica em revistas científicas, livros técnicos e publicações de órgãos oficiais dos últimos 20 anos. Utilizaram-se as bases de dados Lilacs, SciELo e Medline em português, inglês e espanhol, com as palavras-chave: "antioxidantes”, “arteriosclerose”, "dislipidemias", "peroxidação de lipídeos”, "infância”, "vitamina A", “vitamina C” e “vitamina E”.

Síntese de dados: A prevalência de dislipidemia na infância e na adolescência mostra frequência crescente, provavelmente relacionada às mudanças dos hábitos alimentares e à redução na prática de atividades físicas. O elevado nível plasmático da lipoproteína de baixa densidade (LDL-c) é fator de risco para o desenvolvimento da arteriosclerose. O consumo de frutas, verduras e legumes, ricos em antioxidantes, é um dos fatores de maior importância na prevenção da peroxidação lipídica. A baixa ingestão dessas fontes naturais de antioxidantes sugere a necessidade de intervenção nutricional para atingir as metas diárias de consumo de vitaminas $\mathrm{A}, \mathrm{C}$ e E, não sendo preconizada a sua suplementação medicamentosa.

Conclusões: $\mathrm{O}$ pediatra e o nutricionista devem orientar as famílias sobre o consumo de alimentos saudáveis, principalmente frutas, verduras e legumes, por seu potencial efeito antioxidante, especialmente nos primeiros anos de vida.
Palavras-chave: antioxidantes; arteriosclerose; dislipidemias; peroxidação de lipídeos; criança.

\section{ABSTRACT}

Objective: To review the potential antioxidant effects of vitamins $\mathrm{A}, \mathrm{C}$ and $\mathrm{E}$ in the prevention of atherosclerosis development during childhood, emphasizing the prevention of dyslipidemia.

Data sources: Bibliographic search in scientific journals, technical books and official publications of the last 20 years. Lilacs, SciElo and Medline databases were searched for articles in Portuguese, Spanish and English using a combination of the following terms: "antioxidants", "atherosclerosis", "dyslipidemia”, "lipid peroxidation”, "childhood”, "vitamin A", "vitamin C" and "vitamin E".

Data synthesis: There is an increasing prevalence of dyslipidemia in children and adolescents, probably related to changes in dietary habits and to the reduced practice of physical activities. The high plasma concentration of low-density lipoprotein (LDL-c) is a risk factor for atherosclerosis development. The consumption of nutrients rich in antioxidants, such as fruits and vegetables, is very important to prevent lipid peroxidation. The low intake of these natural antioxidants sources suggests the need for nutritional intervention to achieve the daily ingestion targets of vitamins A, C and E. Vitamin supplementation is not recommended.
Instituição: Disciplina de Nutrologia do Departamento de Pediatria da Escola Paulista de Medicina da Universidade Federal de São Paulo (Unifesp), São Paulo, SP, Brasil

${ }^{1}$ Especializanda do Curso de Saúde, Nutrição e Alimentação Infantil da Disciplina de Nutrologia do Departamento de Pediatria da Unifesp, São Paulo, SP, Brasil

${ }^{2}$ Mestranda do Programa de Pós-Graduação em Ciências da Nutrição da Unifesp, São Paulo, SP, Brasil

${ }^{3}$ Doutoranda em Ciências da Nutrição da Unifesp, São Paulo, SP, Brasil

${ }^{4}$ Doutora em Pediatria pelo Departamento de Pediatria da Unifesp; Chefe do Setor de Suporte Nutricional da Disciplina de Nutrologia do Departamento de Pediatria da Unifesp, São Paulo, SP, Brasil
Endereço para correspondência:

Fernanda Luisa Ceragioli Oliveira

Rua Loefgreen, 1.647 - Vila Clementino

CEP 04040-032 - São Paulo/SP

E-mail: fernandalco.dped@epm.br

Conflitos de interesse: nada a declarar

Recebido em: 15/4/2009

Aprovado em: 19/8/2009 
Conclusions: Children should be encouraged to increase ingestion of fruits and vegetables due to their potential antioxidant effect, especially in the first years of life.

Key-words: antioxidants; arteriosclerosis ; dyslipidemias; lipid peroxidation; child.

\section{Introdução}

A prevalência de dislipidemias na infância e na adolescência tem aumentado progressivamente ao longo dos anos, principalmente nos países que sofreram "ocidentalização" dos hábitos de vida ${ }^{(1)}$. Assim, as dislipidemias podem se iniciar na infância, sendo mais frequentes quando as mudanças nos hábitos alimentares associam-se à redução na prática de atividades físicas, com estabelecimento de vida sedentária ${ }^{(2)}$. Há também a determinação genética para desenvolver dislipidemia - nesse caso, chamada de dislipidemia primária ${ }^{(3)}$, sendo as modificações do metabolismo lipídico geradas pela herança familiar. Estas sofrem também influência dos fatores ambientais, como a dieta e a prática de atividade física ${ }^{(4)}$.

A presença de dislipidemia na infância pode influir na velocidade de instalação da aterosclerose ${ }^{(5)}$. Os níveis plasmáticos elevados da lipoproteína de baixa densidade (LDL-c) constituem fator de risco para o desenvolvimento da aterosclerose, pois resultam em aumento da permeabilidade da camada íntima arterial, favorecendo a oxidação da lipoproteína ${ }^{(4,6)}$.

O interesse pelo estilo de vida saudável surge como estratégia para o tratamento e a prevenção dos eventos cardiovasculares $^{(7)}$. Um dos fatores de maior importância para prevenir a peroxidação lipídica e a aterosclerose é a ingestão de antioxidantes, pois esses nutrientes determinam a composição do LDL-c e, consequentemente, sua suscetibilidade à oxidação. Assim, o consumo alimentar é importante determinante de risco cardiovascular, estando a ingestão de frutas, verduras e legumes (ricos em antioxidantes) associada à redução desse risco ${ }^{(8)}$. A terapia nutricional é indicada na prevenção e no tratamento das dislipidemias e deve contemplar as questões culturais, regionais, sociais e econômicas ${ }^{(9)}$.

O objetivo do presente trabalho foi revisar os potenciais efeitos antioxidantes das vitaminas A, C e E para prevenir a propagação da arteriosclerose na infância, com ênfase na prevenção da dislipidemia. Realizou-se pesquisa bibliográfica em revistas científicas, livros técnicos e publicações de órgãos oficiais. Utilizaram-se artigos das bases de dados Lilacs, SiELO e Medline, publicados nos últimos 20 anos, a respeito dos temas dislipidemias na infância, arteriosclerose, antioxidantes (vitaminas A, C e E) e suas funções, em português, inglês e espanhol.

\section{Dislipidemia na infância}

No Brasil, a prevalência de dislipidemia situa-se entre 28 e $40 \%$ das crianças e adolescentes - quando o critério adotado é o de colesterol total (CT) sérico superior a $170 \mathrm{mg} / \mathrm{dL}$ - podendo ser subestimada ao se considerar como valor desejável $<150 \mathrm{mg} / \mathrm{dL}$, proposto pela I Diretriz Brasileira de Prevenção da Aterosclerose na Infância e na Adolescência ${ }^{(5,10)}$.

Na última década, a dislipidemia estendeu-se à faixa pediátrica, quando o início da sequência fisiopatogênica da arteriosclerose passou a ser verificado desde as fases precoces da vida, com a formação de estrias gordurosas precursoras das placas ateroscleróticas. Esse processo tende a ser potencializado no decorrer da vida pela obesidade e outros fatores, como histórico familiar, inatividade física, tipo de dieta e hipertensão $\operatorname{arterial}^{(2,5,11)}$.

Avaliando-se 1.600 escolares entre sete e 14 anos, em Campinas, São Paulo ${ }^{(10)}$, encontrou-se prevalência de $35 \%$ de hipercolesterolemia, considerando-se o valor de corte desejável se $\leq 170 \mathrm{mg} / \mathrm{dL}$. O aumento da prevalência da dislipidemia pode ser observado em estudos nacionais. Avaliação realizada em Bento Gonçalves, Rio Grande do $\mathrm{Sul}^{(12)}$, envolvendo crianças e adolescentes, registrou $28 \%$ de dislipidemia, sendo que $10 \%$ apresentavam aumento do LDL-c. Em outro estudo, realizado em Campina Grande, Paraíba ${ }^{(11)}$, encontrou-se $67 \%$ de dislipidemia em adolescentes de 14 a 17 anos.

\section{Arteriosclerose}

A arteriosclerose é uma doença inflamatória crônica, de origem multifatorial, que ocorre em resposta à agressão endotelial, acometendo principalmente a camada íntima de artérias de médio e grande calibre ${ }^{(9)}$. Diversos estudos têm demonstrado que a doença aterosclerótica da camada íntima/média arterial surge na infância, de forma silenciosa, progredindo significativamente a partir da terceira década de vida $^{(1-3)}$. De acordo com o Bogalusa Heart Study, cerca de 50\% das crianças com um ano apresentam lesões ateromatosas na aorta e, a partir dos dez anos, $100 \%$ apresentam estrias 
gordurosas na aorta e no leito coronário, relacionadas aos níveis séricos de LDL-c $c^{(13)}$.

A formação da placa aterosclerótica inicia-se com a agressão do endotélio vascular por diversos fatores de risco, como a elevação do LDL-c. Consequentemente, essa disfunção endotelial aumenta a permeabilidade da camada íntima às lipoproteínas plasmáticas, favorecendo sua retenção no espaço subendotelial. Retidas, as partículas de LDL-c sofrem oxidação. Outra consequência da disfunção endotelial é o surgimento de moléculas de adesão leucocitária na superfície endotelial, estimulado pela presença de LDL-c oxidada. Assim, os linfócitos e monócitos são atraídos, sendo que os últimos migram para o espaço subendotelial, aonde se diferenciam em macrófagos e captam o LDL-c oxidado, sendo, então, chamados de células espumosas. Estas medem o recrutamento e a proliferação de células musculares lisas e potencializam a inflamação. $\mathrm{O}$ acúmulo de células musculares lisas e a geração de células espumosas, juntamente com outros fatores, são o início da arteriosclerose $\mathrm{e}^{(9,14)}$.

A peroxidação lipídica acontece quando o ácido graxo poli-insaturado (PUFA, do inglês polynsaturated fatty acids), presente na membrana, perde hidrogênio por meio da reação com os radicais livres, caracterizando o estresse oxidativo ${ }^{(14,15)}$. Este tem sido definido como um distúrbio no estado de equilíbrio, no sistema de pró-oxidantes e antioxidantes nas células ${ }^{(16)}$. O conteúdo de antioxidantes da partícula LDL-c é crucial para sua proteção, pois a oxidação do LDL-c somente se inicia após o estresse oxidativo depletar o conteúdo antioxidante celular ${ }^{(6)}$

A prevenção da dislipidemia e, consequentemente, da aterosclerose deve ser iniciada desde o nascimento por meio do aleitamento materno - pois, aparentemente, os níveis mais elevados de colesterol nos lactentes podem induzir a uma regulação hepática do metabolismo dos lipídios ao longo da vida - e ser sustentada por meio de hábitos alimentares saudáveis ${ }^{(5,17)}$.

A prática de atividade física é imprescindível no controle do colesterol. Vale lembrar que o tempo gasto com atividades de lazer junto com a família, nos primeiros anos de vida, é a primeira oportunidade de realizar atividade física e promove motivação suficiente para que a criança valorize tais atividades durante toda a vida, ou seja, o estímulo começa em casa. O tempo de inatividade deve ser limitado a menos de duas horas de televisão/dia, pois esta influencia a dieta das crianças, levando a um consumo excessivo de alimentos com alto teor de sal, carboidrato simples, gordura saturada e $\operatorname{trans}^{(17)}$.

\section{Terapia nutricional na prevenção da arteriosclerose}

Nos últimos anos, observa-se substancial interesse por estratégias terapêuticas de prevenção e tratamento das doenças cardíacas. A intervenção dietética é a melhor escolha para prevenir e tratar as dislipidemias, respeitando-se as necessidades diárias de nutrientes de cada faixa etária ${ }^{(17)}$.

Muitos estudos indicam que o consumo de frutas e vegetais, ricos em substâncias antioxidantes, está associado à redução de 2,6 vezes no risco de doenças cardiovasculares $(\mathrm{DCV})^{(18-22)}$. Estudos com pré-escolares realizado no Rio de Janeiro $^{(23)}$ e em escolares de Florianópolis ${ }^{(24)}$ encontraram inadequação no consumo de frutas e vegetais, utilizando-se como referência a pirâmide alimentar brasileira.

\section{Vitaminas antioxidantes}

Antioxidantes são substâncias capazes de prevenir os efeitos deletérios da oxidação, inibindo o início da peroxidação lipídica e sequestrando radicais livres ${ }^{(25)}$. A quantidade relativa de antioxidantes e pró-oxidantes na dieta influencia a suscetibilidade de um indivíduo desenvolver o estresse oxidativo, que pode ser causado pelo desbalanço nutricional devido à deficiência de antioxidantes e excessiva quantidade de pró-oxidantes ${ }^{(26)}$. Populações com dietas ricas em substâncias antioxidantes apresentam baixa incidência de arteriosclerose coronária, pois os antioxidantes aumentam a resistência do LDL-c à oxidação ${ }^{(7,27,28)}$. O efeito antioxidante das vitaminas A, C e E pode ser mensurado por meio de observações da concentração de lipídeos e da gênese das $\mathrm{DCV}^{(18-22,26)}$.

$\mathrm{O}$ consumo inadequado de vitamina $\mathrm{E}$ relaciona-se ao desenvolvimento e à progressão das doenças crônicas nãotransmissíveis ${ }^{(29)}$. A vitamina $\mathrm{E}$ consiste em um grupo de oito compostos lipossolúveis, cada um dos quais com atividades biológicas específicas, sendo o $\alpha$-tocoferol o mais potente ${ }^{(14)}$ e o mais abundante antioxidante lipossolúvel nos tecidos, plasma e no LDL- ${ }^{(8)}$. O $\alpha$-tocoferol e outras formas de vitamina $\mathrm{E}$ atuam por chain-breaking, pois previnem a oxidação por capturarem os radicais livres e promoverem a primeira linha de proteção contra a peroxidação lipídica ${ }^{(30)}$.

Após a absorção intestinal da vitamina E, esta é incorporada aos quilomícrons. Por meio da ação da lipase lipoproteica (LPL), parte dos tocoferóis transportados nos quilomícrons é captada pelos tecidos extra-hepáticos, e os quilomícrons remanescentes transportam o restante dos tocoferóis ao fígado, onde, pela ação da proteína transportadora de $\alpha$-tocoferol 
( $\alpha$-TTP), a maior parte deste é incorporada nas lipoproteínas de muito baixa densidade (VLDL-c) $)^{(15,16,31)}$.

O conteúdo de vitamina $\mathrm{E}$ determina a suscetibilidade das membranas da LDL-c sofrerem com o dano provocado pelos radicais livres. Estes produzem intermediários secundários, os hidroperóxidos lipídicos, que podem ser decompostos em radicais peroxila e alcoxila, levando a uma cadeia ininterrupta de reações de peroxidação lipídica ${ }^{(16,30)}$. A ação antioxidante da vitamina $E$ é explicada pelo fato de fornecer átomos de hidrogênio para as membranas celulares, impedindo a reação em cadeia que se propaga nas membranas lipídicas. Estudos indicam que a vitamina E pode modular a arteriosclerose por outros mecanismos, incluindo a inibição da adesão e a redução do dano oxidativo de células imunes e endoteliais, além de inibir a proliferação de células musculares lisas ${ }^{(14)}$.

Estudo realizado no norte da Finlândia ${ }^{(32)}$, que se caracteriza pela baixa mortalidade por DCV, comparou os níveis plasmáticos de $\boldsymbol{\alpha}$-tocoferol e demais antioxidantes dessa população com a de outra região, mostrando correlação inversa entre níveis plasmáticos de $\alpha$-tocoferol e mortalidade por DCV. Também houve forte associação entre o consumo de vitamina E e a prevenção de DCV em estudo coorte que analisou o consumo dessa vitamina em adultos inicialmente sem histórico de $\mathrm{DCV}^{(19)}$. Outro estudo transversal avaliou os níveis plasmáticos de vitamina E em crianças com histórico familiar de DCV precoce e naquelas sem esse antecedente familiar, encontrando maior prevalência de deficiência de vitamina E e perfil lipídico alterado no primeiro grupo ${ }^{(21)}$.

A vitamina $\mathrm{E}$ ocorre naturalmente em alimentos de origem vegetal, principalmente nos vegetais verde-escuros, nas sementes oleaginosas, nos óleos vegetais e no germe de trigo, além de estar presente também em alimentos de origem animal, como gema de ovo e fígado ${ }^{(29)}$. A recomendação de consumo da vitamina E, de acordo com as Dietary Reference Intakes (DRI), estabelecidas em 2000, preconiza que a Recommended Dietary Allowance (RDA) leve em conta exclusivamente o $\alpha$-tocoferol, com a finalidade de manter sua concentração plasmática ${ }^{(33)}$ (Tabela 1$)$.

A vitamina A, por sua vez, é o retinol e todos os carotenoides dietéticos com atividade biológica de transretinol. Um dos carotenoides mais estudados é o $\beta$-caroteno, considerado o precursor da vitamina A (pró-vitamina $A)^{(6,8,34)}$. No lúmen intestinal, a vitamina A e os carotenoides emulsificam-se com os sais biliares formando micelas que facilitam a hidrólise dos ésteres de retinil. Tanto a vitamina A ingerida como
Tabela 1 - Ingestão recomendada diária de vitamina E para a faixa etária de zero a 18 anos

\begin{tabular}{lcc}
\hline Faixa etária & RDA/Al * $\left.^{*} \mathbf{m g} / \mathbf{d}\right)$ & UL $^{* *}$ \\
\hline 0 a 6 meses & $4^{*}$ & sem determinação \\
7 a 12 meses & $6^{*}$ & sem determinação \\
1 a 3 anos & 6 & 200 \\
4 a 8 anos & 7 & 300 \\
9 a 13 anos & 11 & 600 \\
14 a 18 anos & 15 & 800 \\
\hline
\end{tabular}

* $\mathrm{Al}$ (Adequate Intake): valor de consumo recomendável baseado em levantamentos, determinações ou aproximações de dados experimentais. É utilizada quando a Recommended Dietary Allowance (RDA) não pode ser determinada; ${ }^{* *}$ UL (Tolerable Upper Intake Level): o mais alto nível de ingestão de um nutriente que não causará efeitos adversos à saúde.

a formada a partir de pró-vitamina esterificam-se com os PUFAs e incorporam-se aos quilomícrons ${ }^{(31)}$.

O mecanismo de ação dos carotenoides como antioxidantes ainda não está bem determinado; porém, acredita-se na sua capacidade de interagir com os radicais livres e espécies reativas de oxigênio ${ }^{(35)}$. Os carotenoides são importantes sequestradores de radicais de oxigênio singlet, interrompendo a geração de carotenoides reativos ao oxigênio ainda nas etapas iniciais de sua formação. Uma única molécula de retinol ou $\beta$-caroteno é capaz de inativar vários radicais de oxigênio singlet. $\mathrm{O} \beta$-caroteno é ainda reconhecido como varredor de radicais do tipo peroxil, interferindo diretamente na reação em cadeia da peroxidação lipídica ${ }^{(26,34)}$. Apesar desses efeitos, os resultados dos estudos de sua associação com DCV são controversos. Em alguns, os níveis de vitamina $A$ não são diferentes em grupos com e sem histórico familiar positivo de evento DCV precoce ${ }^{(21)}$. Em outros, demonstra-se o efeito positivo entre o consumo de $\beta$-caroteno e a inibição da oxidação lipídica ${ }^{(36)}$.

As principais fontes de carotenoides são os legumes, as frutas vermelhas e amarelas e as verduras verdes. $O \beta$-caroteno é encontrado em abundância em abóboras, cenoura, brócolis, almeirão e couve-manteiga ${ }^{(37,38)}$. As novas recomendações de vitamina A são expressas em retinol activity equivalents (RAE) e os carotenoides devem ser convertidos ${ }^{(38)}$ (Tabela 2).

Finalmente, a vitamina $\mathrm{C}$ é uma importante vitamina hidrossolúvel, também conhecida como ácido ascórbico ou ácido desidroascórbico (DHAA) $)^{(22,38)}$, em sua outra forma biologicamente ativa. $\mathrm{O}$ ácido ascórbico é facilmente absorvido a partir do intestino delgado por processo ativo dependente de sódio e por difusão passiva. A eficiência da absorção entérica da vitamina é alta (80-90\%) quando a ingestão é baixa, mas declina marcadamente com 
Tabela 2 - Ingestão recomendada diária de vitamina A para a faixa etária de 0 a 18 anos

\begin{tabular}{|c|c|c|c|c|}
\hline \multirow[t]{2}{*}{ Faixa etária } & \multicolumn{2}{|c|}{ RDA/AI * } & \multicolumn{2}{|c|}{$\mathrm{UL}^{* *}$} \\
\hline & (mcg RAE**/dia) & (mcg $\beta$-caroteno/dia) & (mcg RAE $\left.{ }^{* * *} / \mathrm{dia}\right)$ & (mcg $\beta$-caroteno/dia) \\
\hline 0 a 6 meses & 400 & 4800 & 600 & 7200 \\
\hline 7 a 12 meses & 500 & 6000 & 600 & 7200 \\
\hline 1 a 3 anos & 300 & 3600 & 600 & 7200 \\
\hline 4 a 8 anos & 400 & 4800 & 900 & 10800 \\
\hline 9 a 13 anos & 600 & 7200 & 1700 & 20400 \\
\hline 14 a 18 anos & o 700 & q 8400 & q 2800 & 33600 \\
\hline & 1 900 & 10800 & 2800 & 33600 \\
\hline
\end{tabular}

${ }^{*} \mathrm{Al}$ (Adequate Intake): valor de consumo recomendável baseado em levantamentos, determinações ou aproximações de dados experimentais. É utilizada quando a Recommended Dietary Allowance (RDA) não pode ser determinada; ** UL (Tolerable Upper Intake Level): é o mais alto nível de ingestão de um nutriente que não causará efeitos adversos à saúde. ${ }^{* *}$ RAE (Retinol Activity Equivalents): 1 RAE=12mcg de $\beta$ - caroteno; 1 $\operatorname{RE}($ retinol $)=6 \mathrm{mcg}$ de $\beta$-caroteno; $1 \mathrm{mcg}$ de $\beta$-caroteno=0,167 mcg de retinol; 1 Unidade Internacional (UI) de vitamina $A=0,3 \mathrm{mcg}$ de retinol.

o aumento da ingestão(38). A vitamina C é transportada no plasma na forma de ácido ascórbico em solução livre, sendo capturada pelas células do sistema transportador de glicose e pelo sistema de transporte ativo específico. Cada sistema mobiliza o ácido desidroascórbico para dentro das células, onde é reduzido a ascorbato ${ }^{(39)}$. O ácido ascórbico é considerado o mais importante e potente antioxidante nutricional hidrossolúvel ${ }^{(40)}$.

Acredita-se que o ácido ascórbico proteja contra a peroxidação lipídica de duas maneiras. Primeiro, de forma direta, eliminando os radicais peróxidos antes que eles iniciem a peroxidação lipídica. No plasma, a vitamina C atua como agente redutor, doando elétrons para várias espécies reativas, eliminando-as antes que elas reajam com as membranas e as lipoproteínas ${ }^{(7)}$. Segundo, de modo indireto, a vitamina $\mathrm{C}$ regenera a forma ativa da vitamina $\mathrm{E}$ e de outros antioxidantes como o $\beta$-caroteno, flavonoides e glutationa para que exerçam seu papel antioxidante ${ }^{(6)}$. Além disso, o ácido ascórbico parece interferir sobre outros fatores relacionados ao risco cardiovascular, como a integridade do tecido vascular, o tônus vascular, o metabolismo lipídico e a pressão arterial ${ }^{(7)}$. Dessa forma, uma dieta que inclua alimentos fontes de vitamina $\mathrm{C}$ como frutas cítricas, frutas vermelhas, tomate e brócolis seria benéfica, sem os riscos acarretados por megadoses dessa vitamina ${ }^{(38)}$. Uma ingestão regular e adequada é preconizada em virtude da capacidade de armazenamento dessa vitamina no organismo e em decorrência de sua constante utilização. Sendo assim, deve-se atender às recomendações preconizadas pelas $\mathrm{DRI}^{(37)}$ (Tabela 3).

Em 1991, o estudo Multinational Monitoring of Trends and Determinants in Cardiovascular Disease (MONICA), realizado em populações europeias, mostrou significante associação
Tabela 3 - Ingestão recomendada diária de vitamina C, para a faixa etária de zero a 18 anos

\begin{tabular}{lcc}
\hline Faixa etária & RDAlAl $^{*}(\mathbf{m g} / \mathbf{d})$ & UL $^{* *}$ \\
\hline 0 a 6 meses & 40 & sem determinação \\
7 a 12 meses & 50 & sem determinação \\
1 a 3 anos & 15 & 400 \\
4 a 8 anos & 25 & 650 \\
9 a 13 anos & 45 & 1200 \\
14 a 18 anos & $0: 75$ & $0: 1.800$ \\
& $+: 65$ & $+: 1.800$ \\
\hline
\end{tabular}

* $\mathrm{Al}$ (Adequate Intake): valor de consumo recomendável baseado em levantamentos, determinações ou aproximações de dados experimentais. É utilizada quando a Recommended Dietary Allowance (RDA) não pode ser determinada; ** UL (Tolerable Upper Intake Level): é o mais alto nível de ingestão de um nutriente que não causará efeitos adversos à saúde.

inversa entre níveis plasmáticos de vitamina $\mathrm{C}$ e mortalidade por $\mathrm{DCV}^{(41)}$. Pesquisa realizada em crianças com histórico familiar de DCV precoce mostrou que estas apresentavam níveis plasmáticos de vitamina $\mathrm{C}$ adequados ${ }^{(21)}$. Vale alertar que, hoje em dia, grande parte do consumo diário de vitamina $C$ deve-se à elevada ingestão de sucos artificiais enriquecidos com essa vitamina, que possuem alto teor de açúcares e substâncias químicas e não são recomendados em uma dieta saudável ${ }^{(24)}$.

\section{Suplementação medicamentosas de vitaminas antioxidantes}

Com a justificativa de que as DRI para essas vitaminas são determinadas apenas como a quantidade necessária para evitar a sua deficiência, não levando em consideração seus efeitos antioxidantes, alguns autores defendem a ideia de utilizar a suplementação medicamentosa dessas 
vitaminas, principalmente da vitamina $\mathrm{E}$, para garantir o efeito antioxidante ${ }^{(19,30)}$.

Vários estudos descrevem os efeitos da ingestão de doses de vitamina $\mathrm{E}$ acima das estabelecidas pela atual recomendação, na forma de suplementos, na prevenção de doenças crônicas não-transmissíveis e no estímulo do sistema imune ${ }^{(18,42)}$. Em contrapartida, Guinazi ${ }^{(43)}$ relata que a atual recomendação de vitamina E levaria em consideração a atuação dessa vitamina na prevenção de doenças relacionadas ao estresse oxidativo e que altas doses de vitamina E estariam associadas a um efeito pró-oxidante ${ }^{(44,45)}$.

Pesquisa espanhola ${ }^{(45)}$ randomizou 40 crianças portadoras de hipercolesterolemia familiar heterozigótica (LDL-c $>160 \mathrm{mg} / \mathrm{dL}$ ), com média de idade de dez anos, em dois grupos: o primeiro recebeu terapia com vitamina $C$ (1000mg/dia) e vitamina E (400UI/dia) e, ao segundo, não foi ofertada suplementação vitamínica. O resultado mostrou não haver diferença significativa no perfil lipídico plasmático das crianças. Em contraste, outro estudo realizado com 15 pacientes de nove a 20 anos com hipercolesterolemia familiar (LDL-c $>130 \mathrm{mg} / \mathrm{dL}$ ) utilizou suplementos de vitamina E (400UI/dia) e C (500mg/dia) por seis semanas ${ }^{(42)}$. Os autores observaram restauração da função endotelial em crianças dislipidêmicas com a suplementação medicamentosa e concluíram ser a suplementação fundamental para crianças com dislipidemia, pois apenas $20 \%$ delas consomem cinco ou mais porções de frutas e hortaliças por dia.

Em relação à suplementação com $\beta$-caroteno, a literatura é escassa. Os poucos estudos de intervenção não encontraram benefícios em relação à prevenção de doença cardiovascular por meio de sua suplementação ${ }^{(46,47)}$.

Meta-análise de sete estudos controlados, duplo-cegos e randomizados não mostrou evidências de que os suplementos de antioxidantes (vitamina C, E e $\beta$-caroteno) previnam a progressão da arteriosclerose em adultos ${ }^{(48)}$. Outra meta-análise ${ }^{(19)}$ com sete estudos do tipo coorte, que relacionaram a ingestão de vitaminas antioxidantes e a sua suplementação com as doenças cardiovasculares em adultos, mostrou que a suplementação de vitamina $C$ (mediana de ingestão + suplementação de $756 \mathrm{mg} /$ dia) obteve resultado positivo quanto à prevenção de doença cardiovascular. Em relação à suplementação com vitamina $\mathrm{E}$ e $\beta$-caroteno, não houve benefícios.

Muitas questões importantes devem ser respondidas antes de se recomendar, de forma universal, a suplementação de vitaminas antioxidantes. A principal questão relaciona-se à toxicidade. Quanto ao $\alpha$-tocoferol e $\beta$-caroteno, parece não existir efeito colateral com seu consumo elevado, pois não são substâncias carcinogênicas, mutagênicas ou teratogênicas. Entretanto, não é recomendada a suplementação de $\alpha$-tocoferol em pacientes em terapia anticoagulante ou com doença autoimune, pois pode precipitar acidentes hemorrágicos ou desencadear surtos autoimunes ${ }^{(16,49)}$.

Não há estudos randomizados, controlados e com número suficiente de pacientes pediátricos ou adultos que demonstrem a prevenção de eventos relacionados à aterosclerose por meio da suplementação medicamentosa com antioxidantes. Portanto, não havendo tal evidência, a suplementação não é recomendada pela IV Diretriz Brasileira sobre Dislipidemias e Prevenção da Aterosclerose de $2007^{(9)}$ e pela American Heart Association, que recomenda o consumo de dieta balanceada com frutas, verduras e legumes ${ }^{(9)}$.

\section{Conclusão}

Percebe-se tendência mundial no aumento da prevalência das dislipidemias na infância, decorrentes de fatores ambientais e familiares, aliada ao fato de o processo arteriosclerótico ser detectado cada vez mais precocemente na vida, observando-se a presença de estrias gordurosas em fetos de mães com dislipidemia. $\mathrm{O}$ aumento da prevalência da dislipidemia está fortemente associado às mudanças na alimentação, com predomínio de alimentos industrializados, que contribuem para a alteração do perfil lipídico e menor consumo de alimentos in natura, fontes de vitaminas antioxidantes. Muitas famílias têm buscado soluções rápidas para se alimentar, consumindo excessivamente os produtos prontos (industrializados) e fast-foods. Desse modo, a criança perde o referencial do que seria uma alimentação saudável. Torna-se fundamental que toda a família conheça os benefícios do consumo de alimentos in natura, como frutas e legumes, e que os incorporem em sua alimentação. Vale lembrar que, embora o fator genético também influencie a suscetibilidade da criança para desenvolver um processo arteriosclerótico, suas manifestações clínico-metabólicas podem ser potencializadas ou amenizadas pelos fatores ambientais.

Em pleno século 21, imagina-se que a recomendação de promoção de um estilo de vida saudável visando à prevenção de doenças crônicas não-transmissíveis seja conceito préadquirido pelo profissional da saúde e, consequentemente, pela população. Entretanto, o levantamento dos dados mostra que hábitos alimentares e estilo de vida inapropriados para 
a prevenção de DCV na infância e na adolescência ainda são frequentes. Assim, o pediatra e o nutricionista devem indicar às famílias o consumo de alimentos saudáveis, principalmente frutas, verduras e legumes, por seu potencial efeito antioxidante. Além disso, os profissionais de saúde devem reforçar a necessidade de se reduzir o consumo de alimentos industrializados, principalmente nos primeiros anos de vida. Não se recomenda a suplementação medicamentosa de vitaminas antioxidantes por escassez de evidências clínicas na literatura.

\section{Referências bibliográficas}

1. Giuliano IC, Coutinho MS, Freitas SF, Pires MM, Zunino JN, Ribeiro RQ. Serum lipids in school kids and adolescents from Florianópolis, SC, Brazil - Healthy Floripa 2040 study. Arq Bras Cardiol 2005;85:85-91.

2. Faria EC, Dalpino FB, Takata R. Lípides e lipoproteínas séricos em crianças e adolescentes ambulatoriais de um hospital universitário público. Rev Paul Pediatr 2008;26:54-8.

3. Daniels SR, Greer FR; Committee on Nutrition. Lipid screening and cardiovascular health in childhood. Pediatrics 2008;122:198-208.

4. Giuliano IC, Caramelli B. Dislipidemias em crianças e adolescentes. Rev Soc Cardiol Estado de São Paulo 2005;15:518-23.

5. Giuliano ICB, Caramelli B. Dislipidemias na infância e na adolescência. Pediatria (São Paulo) 2008;29:275-85

6. Batlouni M. Hipótese oxidativa da aterosclerose e emprego dos antioxidantes na doença arterial coronária. Arq Bras Cardiol 1997;68:55-63.

7. Rique $A B$, Soares $E A$, Meirelles $C M$. Nutrição e exercício na prevenção e controle das doenças cardiovasculares. Rev Bras Med Esporte 2002;8:244-54.

8. Duell PB. Prevention of atherosclerosis with dietary antioxidants: fact or fiction? J Nutr 1996;126:1067S-71.

9. Departamento de Aterosclerose da Sociedade Brasileira de Cardiologia. IV Diretriz Brasileira sobre Dislipidemias e Prevenção da Aterosclerose. Arq Bras Cardiol 2007;88:2-19.

10. Moura EC, Castro CM, Mellin AS, Figueiredo DB. Perfil lipídico em escolares de Campinas, SP, Brasil. Rev Saúde Pública 2000;34:499-505.

11. Carvalho DF, PaivaAA, Melo AS, Ramos AT, Medeiros JS, Medeiros CC et al. Perfil lipídico e estado nutricional de adolescentes. Rev Bras Epidemiol 2007;10:491-8.

12. Gerber ZR, Zielinsky P. Fatores de risco de aterosclerose na infância. Um estudo epidemiológico. Arq Bras Cardiol 1997;69:231-6.

13. Berenson GS, Wattigney WA, Tracy RE, Newman WP 3rd, Srinivasan SR, Webber LS et al. Atherosclerosis of the aorta and coronary arteries and cardiovascular risk factors in persons aged 6 to 30 years and studied at necropsy (The Bogalusa Heart Study). Am J Cardiol 1992;70:851-8.

14. Martins CM, Oliveira DM, Teixeira TF, Peluzio MC. O paradoxo do papel da vitamina E na iniciação e progressão da aterosclerose e sua correlação com os radicais livres. Rev Med Minas Gerais 2004;14:113-6.

15. Herrera E, Barbas C. Vitamin E: action, metabolism and perspectives. J Physiol Biochem 2001;57:43-56.

16. Jordão Jr AA, Chiarello PG, Bernardes MS, Vannucchi H. Lipid peroxidation and ethanol: role of vitamin-E and glutathione. Medicina (Ribeirão Preto) 1998;31:434-49.

17. Sociedade Brasileira de Cardiologia. I Diretriz de prevenção da aterosclerose na infância e na adolescência. Arq Bras Cardiol 2005;85:3-36.

18. Ye Z, Song H. Antioxidant vitamins intake and the risk of coronary heart disease: meta-analysis of cohort studies. Eur J Cardiovascular Prev Rehabil 2008;15:26-34.

19. Knekt P, Ritz J, Pereira MA, O’Reilly EJ, Augustsson K, Fraser GE et al. Antioxidant vitamins and coronary heart disease risk: a pooled analysis of 9 cohorts. Am J Clin Nutr 2004;80:1508-20.

20. Gale CR, Ashurst HE, Powers HJ, Martyn CN. Antioxidant vitamin status and carotid atherosclerosis in the elderly. Am J Clin Nutr 2001;74:402-8.

21. Sutil de Naranjo R, Márquez M, Barrios de Jordán M, Briceño de Chemello F, Torres M, Rivas de Yépez CE et al. Niveles de vitaminas antioxidantes de lípidios en escolares con historia familiar de enfermedad arterial coronaria. Arch Venez Farmacol Terap 2003;22:163-71.
22. Cerqueira FM, Medeiros MH, Augusto O. Dietetic antioxidants: controversies and perspectives. Quím Nova 2007;30:441-9.

23. Barbosa RM, Croccia C, Carvalho CG, Franco VC, Salles-Costa R, Soares EA. Consumo alimentar de crianças com base na pirâmide alimentar brasileira infantil. Rev Nutr 2005;18:633-41.

24. Fiates GM, Amboni RD, Teixeira E. Comportamento consumidor, hábitos alimentares e consumo de televisão por escolares de Florianópolis. Rev Nutr 2008;21:105-14.

25. Rodrigues HG, Diniz YS, Faine LA, Almeida JA, Fernandes AA, Novelli EL. Suplementação nutricional com antioxidantes naturais: efeito da rutina na concentração de colesterol - HDL. Rev Nutr 2003;16:315-20.

26. Vannucchi H, Moreira EA, Cunha DF, Junqueira-Franco MV, Bernardes MM, Jordão Jr AA. Role of nutrients on lipid peroxidation and antioxidant defense system. Medicina (Ribeirão Preto) 1998;31:31-44.

27. Cabrera TC, Serrano DS. Algunos aspectos sobre el estrés oxidativo, el estado antioxidante y la terapia de suplementación. Rev Cubana Cardiol 2000;14:55-60

28. Kritharides $\mathrm{L}$, Stocker $\mathrm{R}$. The use of antioxidant supplements in coronary heart disease. Atherosclerosis 2002;164:211-9.

29. Batista ES, Costa AG, Pinheiro-Sant'ana HM. Adição da vitamina E aos alimentos: implicações para os alimentos e para a saúde humana. Rev Nutr 2007;20:525-35

30. Pruthi S, Allison TG, Hensrud DD. Vitamin E supplementation in the prevention of coronary heart disease. Mayo Clin Proc 2001;76:1131-6.

31. Marquez M, Yépez CE, Sútil-Naranjo R, Rincón $M$. Aspectos básicos y determinación de las vitaminas antioxidantes $E$ y A. Invest Clin 2002;43:191-204

32. Luoma PV, Näyhä S, Sikkilä K, Hassi J. High serum alpha-tocopherol, albumin selenium and cholesterol, and low mortality from coronary heart disease in northern Finland. J Intern Med 1995;237:49-54.

33. Amaya-Farfan J, Domene SM, Padovani RM. DRI: síntese comentada das novas propostas sobre recomendações nutricionais para antioxidantes. Rev Nutr 2001;14:71-8.

34. Gomes MM, Saunders C, Accioly E. Vitamin A role preventing oxidative stress in newborns. Rev Bras Saude Matern Infant 2005;5:275-82.

35. Cardoso SL. Fotofísica de carotenóides e o papel antioxidante de $\beta$-caroteno. Química Nova 1997;20:535-40.

36. Lavy A, Ben Amotz A, Aviram M. Preferential inhibition of LDL oxidation by the all-trans isomer of beta-carotene in comparison with 9-cis beta-carotene. Eur J Clin Chem Clin Biochem 1993;31:83-90.

37. Mourão DM, Sales NS, Coelho SB, Pinheiro-Santana HM. Biodisponibilidade de vitaminas lipossolúveis. Rev Nutr 2005;18:529-39.

38. Philippi ST. Pirâmide dos alimentos: fundamentos básicos da nutrição. São Paulo: Manole; 2008.

39. Mahan LK, Escott-Stump S. Krause: alimentos, nutrição e dietoterapia. $11^{\text {nd }}$ ed. São Paulo: Roca; 2005.

40. Sesso HD, Buring JE, Christen WG, Kurth T, Belanger C, MacFadyen $\mathrm{J}$ et al. Vitamins $\mathrm{E}$ and $\mathrm{C}$ in the prevention of cardiovascular disease in men: the Physicians' Health Study II randomized controlled trial. JAMA 2008;300:2123-33.

41. Gey KF, Puska P, Jordan P, Moser UK. Inverse correlation between plasma vitamin $E$ and mortality from ischemic heart disease in cross-cultural epidemiology. Am J Clin Nutr 1991;53 (Suppl 1):326S-34. 
42. Engler MM, Engler MB, Malloy MJ, Chiu EY, Scholoetter MC, Paul SM et al. Antioxidant vitamins $\mathrm{C}$ and $\mathrm{E}$ improve endothelial function in children with hyperlipidemia: Endothelial Assessment of Risk from Lipids in Youth (EARLY) Trial. Circulation 2003;108:1059-63.

43. Guinazi M. Tocoferóis e tocotrienóis em hortaliças, ovos e óleos vegetais utilizados em restaurantes comerciais [dissertação]. Viçosa (MG): UFV; 2004.

44. Chopra M, Thurnham DI. Antioxidants and lipoprotein metabolism. Proc Nutr Soc 1999;58:663-71.

45. Aldámiz-Echevarría L, Dalmau J, Prieto JA, Andrade F, Sanjuro P, Elorz J et al. Ensayo aleatorizado ciego-sencillo sobre los efectos de las vitaminas $\mathrm{C}$ y E en la hipercolesterolemia familiar. An Pediatr (Barc) 2006;65:101-7.
46. Lee IM, Cook NR, Manson JE, Buring JE, Hennekens CH. Beta-carotene supplementation and incidence of cancer and cardiovascular disease: the Women's Health Study. J Natl Cancer Inst 1999;91:2102-6.

47. Hennekens CH, Buring JE, Manson JE, Stampfer M, Rosner B, Cook NR et al. Lack of effect of long-term supplementation with beta carotene on the incidence of malignant neoplasms and cardiovascular disease. N Engl J Med 1996;334:1145-9.

48. Bleys J, Miller ER 3rd, Pastor-Barriuso R, Appel LJ, Guallar E. Vitamin-minera supplementation and the progression of atherosclerosis: a meta-analysis of randomized controlled trials. Am J Clin Nutr 2006;84:880-7.

49. Adams AK, Wermuth EO, McBride PE. Antioxidant vitamins and the prevention of coronary heart disease. Am Fam Physician 1999;60:895-904. 